\title{
Vibration Response of Ultralight Coilable Spacecraft Structures
}

\author{
James Umali* \\ Northrop Grumman Aerospace Systems, Azusa, CA 91702 \\ Lee Wilson ${ }^{\dagger}$ and Sergio Pellegrino ${ }^{\ddagger}$ \\ California Institute of Technology, Pasadena, CA 91125
}

\begin{abstract}
Dynamic assessment of ultralight wrapped spacecraft structures subject to the launch vibration environment is difficult, and has been foregone in many space programs in favor of purely experimental test campaigns. We present a numerical methodology to better understand the wrapped state of a structure and the dynamic behavior during launch vibration, enabling higher confidence in its survivability and greater understanding of the dynamic behavior at different scales, which vibration test approaches alone cannot achieve.
\end{abstract}

\section{Nomenclature}

E Elastic Modulus (GPa)

$\nu \quad$ Poisson's Ratio

$t \quad$ Time $(\mathrm{s})$

\section{Introduction}

Large, ultra-thin spacecraft structures have been proposed for future space-based solar power satellites. ${ }^{1}$ It is envisaged that these structures, consisting of carbon-fiber Triangular And Collapsible (TRAC) longerons supporting elastically collapsible, multi-functional tiles that convert sunlight into microwave power that is beamed to Earth, would be launched in a tightly wrapped configuration. These structures are very different from traditional satellites and their behavior under launch vibrations needs to be understood and quantified to enable this type of missions.

Due to the complexity of these types of structures, current flight qualification methodology is typically focused on a series of dynamic and vibration tests with minimal analytical modeling, simulation, and theoretical work to back up the experimental measurements. ${ }^{2,3}$ Although effective and economical, this approach can only demonstrate the survivability of the as-tested structure, but it provides minimal insight on the dynamic behavior of the structure or the response of the structure at different scales. This introduces significant limitations in the early development and design optimization of large area structures where fabrication of the full-scale model is premature.

In this paper, we present a methodology to quantify the dynamic behavior of wrapped space structures under the launch vibration environment, taking into account an estimation of the shape and pressure distribution in the wrapped configuration. We utilize results from small-scale experimental test models to verify the predictions from a numerical finite element model (FEM), including contact forces and friction between adjacent layers, which at a later stage could be used to make predictions on the dynamic behavior at full scale.

\footnotetext{
${ }^{*}$ Visiting Associate, Caltech; Mechanical Engineer, Northrop Grumman, 1100 W Hollyvale St.

${ }^{\dagger}$ Ph.D. Candidate, Graduate Aerospace Laboratories, MC 205-45, AIAA Student Member.

$\ddagger$ Joyce and Kent Kresa Professor of Aeronautics and Professor of Civil Engineering, Graduate Aerospace Laboratories, MC 301-46, AIAA Fellow.
} 


\section{Coilable Spacecraft Structures}

Several space programs have been developed or are currently in development utilizing large, rollable or coilable, ultralight structures with innovative deployment techniques. An example is the Composite Beam Roll Out Array (COBRA $)^{2,4}$ program by Alliance Space Systems. The Roll-Out Solar Array (ROSA) ${ }^{3,5}$ program developed by Deployable Space Systems features a thin, lightweight solar array blanket supported along the long edges with lightweight structural members that can be rolled and/or deployed in one direction. Such a configuration allows a long narrow solar blanket to be efficiently and compactly packaged along one dimension. The Interplanetary Kite-craft Accelerated by Radiation Of the Sun (IKAROS) program, developed by the Japan Aerospace Exploration Agency (JAXA), was launched in May 2010 and was the first spacecraft to successfully demonstrate solar sail technology in interplanetary space. ${ }^{6,7}$ With size of $14 \mathrm{~m} \times$ $14 \mathrm{~m}$, this planar blanket-like structure required compaction in 2 dimensions for launch. This required the structure to be divided into four right-triangular quadrants with individual strips subdividing each quadrant. For packaging, the strips in each quadrant were Z-folded to create a star-shaped pattern, which was then wound around the center to produce a tightly wrapped roll.

The focus of the present study is to develop a technique to estimate vibration response behavior for a wrapped configuration similar to that of the Space Solar Power Initiative (SSPI), ${ }^{1}$ which uses the packaging concept shown in Figure 1, similar to that used by the IKAROS program. The folding pattern consists of alternating mountain and valley folds between strips, as shown in Figure 1a. After folding as shown in Figure 1b, a star-like shape with four arms (Figure 1c) is obtained. Wrapping these arms (Figure 1d) produces the wrapped configuration shown in Figure 1e.

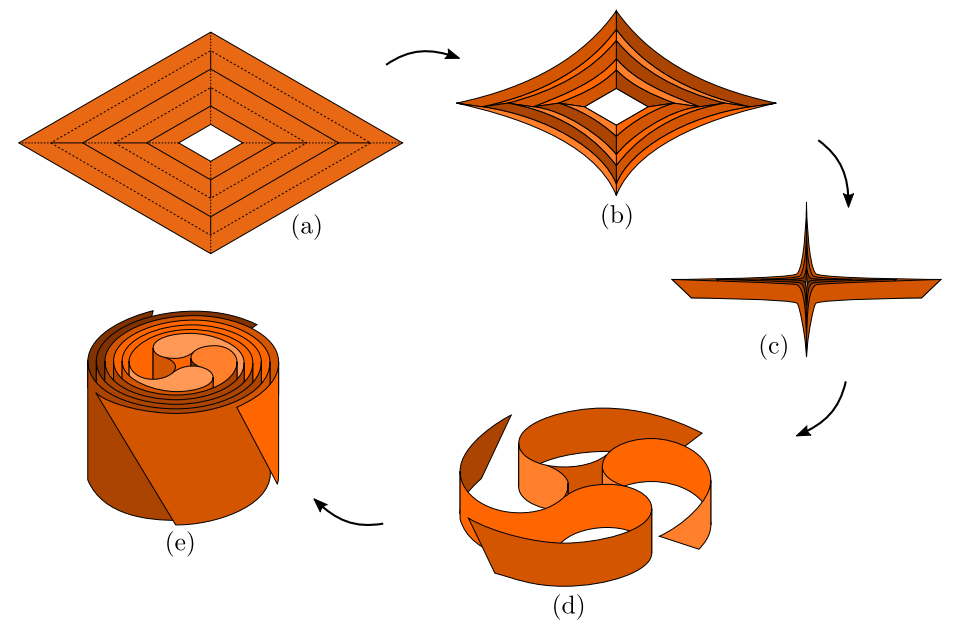

Figure 1. Packaging concept. ${ }^{1}$

Although a rigorous vibration test campaign alone may be sufficient for the design and development of smaller structures, it would be impractical to build and test full-scale prototypes of the nominal 60 $\mathrm{m} \times 60 \mathrm{~m}$ design. Furthermore, the wrapping process introduces co-folding of high-strain composite and delicate photovoltaic components and there is concern that relative movement between strips during launch vibration may potentially damage or degrade the optical quality concentrators on the strips. Hence, numerical simulations are necessary to understand the dynamic behavior of these types of structures during wrapping and launch vibration. An accurate numerical model can ensure adequacy of the launch constraints and structural strength of the system during early design and development of the program.

\section{Experimental Vibration Model}

Traditional methods for estimating random vibration response behavior of standard spacecraft structures primarily involve implementation of built-in FEA random vibration modules. For example, MSC Patran contains a "Patran Random" module allowing users to impose a PSD-vs-Frequency random vibration profile on a structure to directly obtain the root mean square acceleration and stress responses to that profile. However, the algorithms for modal and frequency response analyses that these modules rely on are inherently 


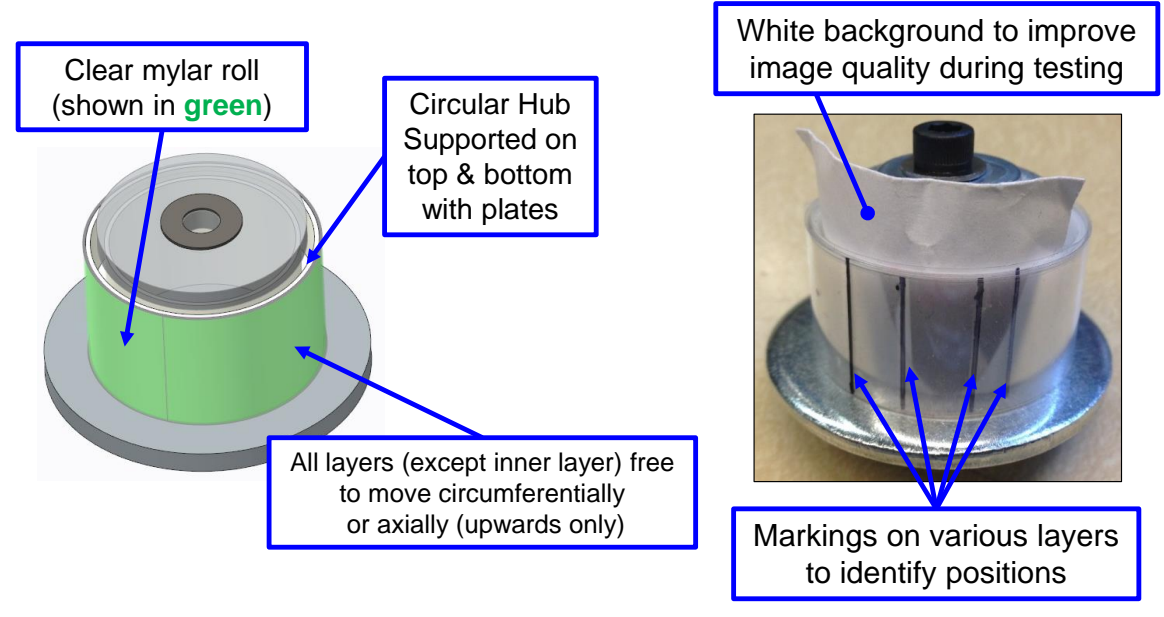

Figure 2. Experimental setup for coiled strip test.

linear and are unable to include non-linear effects like contact or friction. An alternate approach involving a small-scale experimental test model is proposed, which will be used in a later section to develop and validate a non-linear dynamic explicit FEA model.

This section introduces a novel technique to measure the motion of ultralight wrapped structures and demonstrate its application through vibration testing of a simple single-layer roll of clear mylar. The application of this technique on a more refined experimental vibration model utilizing a tensioned roll of Kapton polyimide is then presented.

\section{III.A. Development of Vibration Measurement Technique}

Measuring the motion of ultralight wrapped structures under a vibration environment presents several challenges, preventing the use of conventional measurement techniques. Firstly, due to the structure's lightweight nature, the use of piezoelectric accelerometers directly attached to the structure is not feasible due to their significant contributions in weight, which would affect the resonance frequencies and hence vibration response behavior. Laser vibrometers are a viable option when considering out-of-plane motion (parallel to the laser direction) since they provide a non-contact option for measurement. However, laser vibrometers are unable to measure in-plane motion unless a set of three laser vibrometer scanning heads are used. ${ }^{9}$ Another challenge is identifying the motion of the inner layers of a wrapped structure. While the outermost layer is easily accessible for measurement, inner layers are not visible and hence cannot easily be measured, rendering laser vibrometry unfeasible.

First, to study the effect of sliding between wrapped layers in a vibration environment, we addressed these challenges on a simple model comprising a strip of $0.04 \mathrm{~mm}$-thick transparent mylar loosely wrapped around a circular hub, as shown in Figure 2. The mylar strip was constrained to the hub at one end, and then looped around the hub 10 times to produce a 10-layer roll. The outermost layer was then constrained to the next adjacent inner layer, allowing all layers (except the innermost layer) to move freely in a circumferential direction. A circular plate supported the bottom of the roll, and a circular plate (with a diameter smaller than the roll) supported the top of the hub, allowing the mylar layers to move axially without any constraint in the upwards direction. Vertical, black lines were marked on various layers, e.g. the 1st, 4th, 7th, and 10th layers, as shown in Figure 2 to provide a visual indicator of the relative circumferential positions for each of these layers. These lines may also be horizontal, to provide an indicator of the relative axial positions of the layers, as shown in Figure $6 \mathrm{~b}$. The use of a transparent material is essential to allow this visualization technique.

The mylar roll was subjected to vibration testing using the setup shown in Figure 3a. In this particular setup, a 10-layer mylar roll with horizontal markings on the 1st, 4th, 7th, and 10th layers was used to identify relative axial motion during an axial-direction sinusoidal vibration test at a forcing frequency of $20 \mathrm{~Hz}$. A laser vibrometer was used for reference to measure the base motion response as a function of time, and a high speed camera (recording at $1000 \mathrm{~Hz}$ sampling frequency) was used to record the positions of the black 
markings during the test.

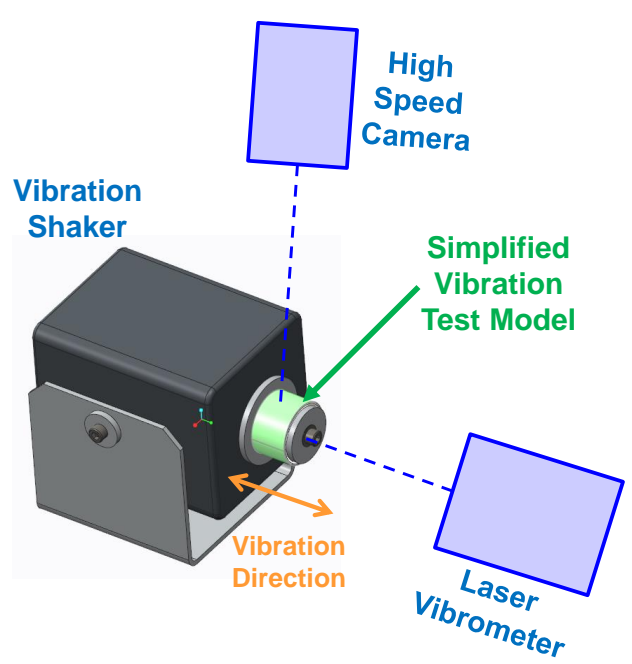

(a) Vibration Test Setup
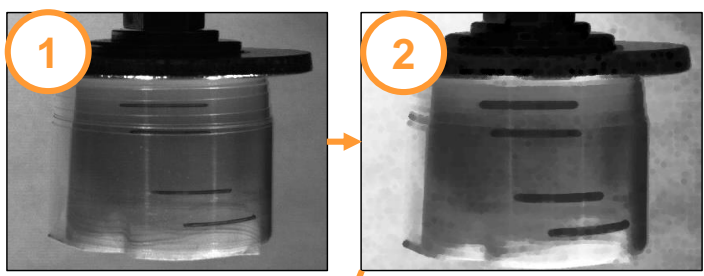

Image "erosion"

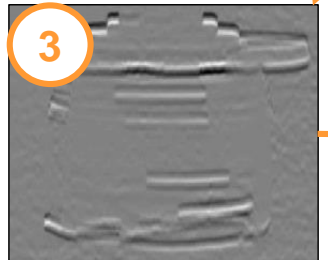

Edge Filtering

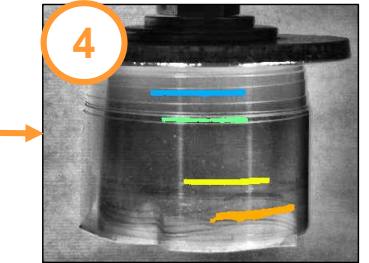

Region Detection

(b) Image Processing Routine

Figure 3. Test setup and image processing routine of mylar roll vibration experiments.

The images were processed in Matlab, as shown in Figure 3b, to automate the marking detection routine. First, the images undergo an "erosion" step which applies a minimum filter in the form of a rolling 3pixel radius ball, which effectively thickens the black lines and removes glare/reflection at the edges of the layers. Edge filtering is achieved by performing a 2-D convolution of the image with a custom-designed mask that would specifically highlight horizontal edges. After application of a resulting brightness criteria and extracting regions of specified length and width requirements, the black markings are successfully detected.

Since the mylar layers were wrapped loosely and since axial motion in one direction is essentially unconstrained, large axial displacements of individual layers were observed during the axial sinusoidal vibration test on the mylar roll, as shown in Figure 4. By processing individual images from the high speed camera, the centroid vertical positions over time for the first $0.5 \mathrm{sec}$ were determined as shown in Figure 5 . A close inspection of Figure 5 indicates that the motion of the outermost layer (in orange, i.e. the bottom-most horizontal line) and the fourth outermost layer (in yellow, i.e. the second horizontal line from the bottom) exhibit some phase delay compared with the other two markings (which correspond to the innermost layer and the fourth innermost layer). Such observations were achievable through the measurement methodology implemented in this experiment, using a thin transparent material, high-speed photography, and image processing techniques.

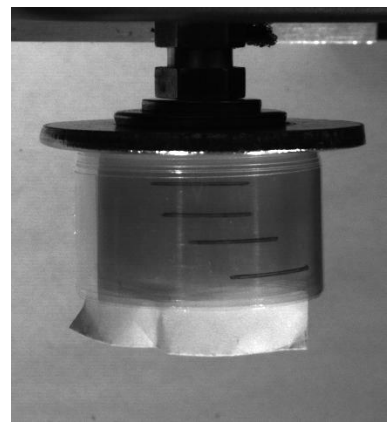

(a) $t=5.787 \mathrm{~s}$

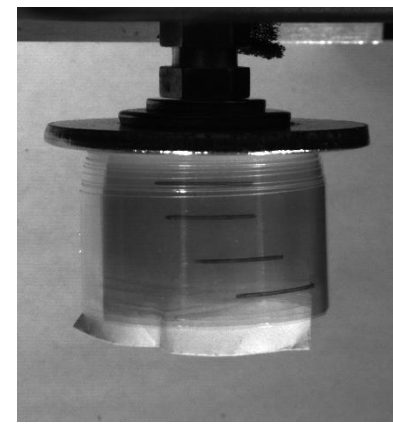

(b) $t=5.795 \mathrm{~s}$

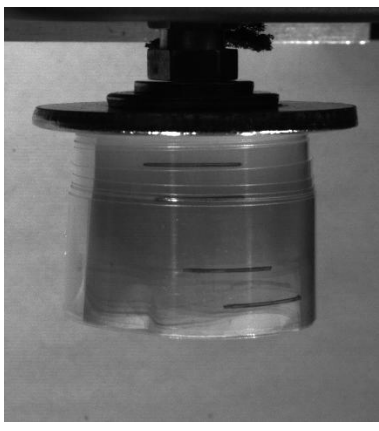

(c) $t=5.801 \mathrm{~s}$

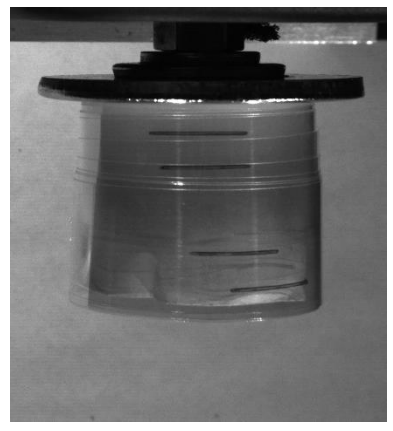

(d) $t=5.810 \mathrm{~s}$

Figure 4. Positions of mylar roll layers at various times during $20 \mathrm{~Hz}$ axial sinusoidal vibration. 

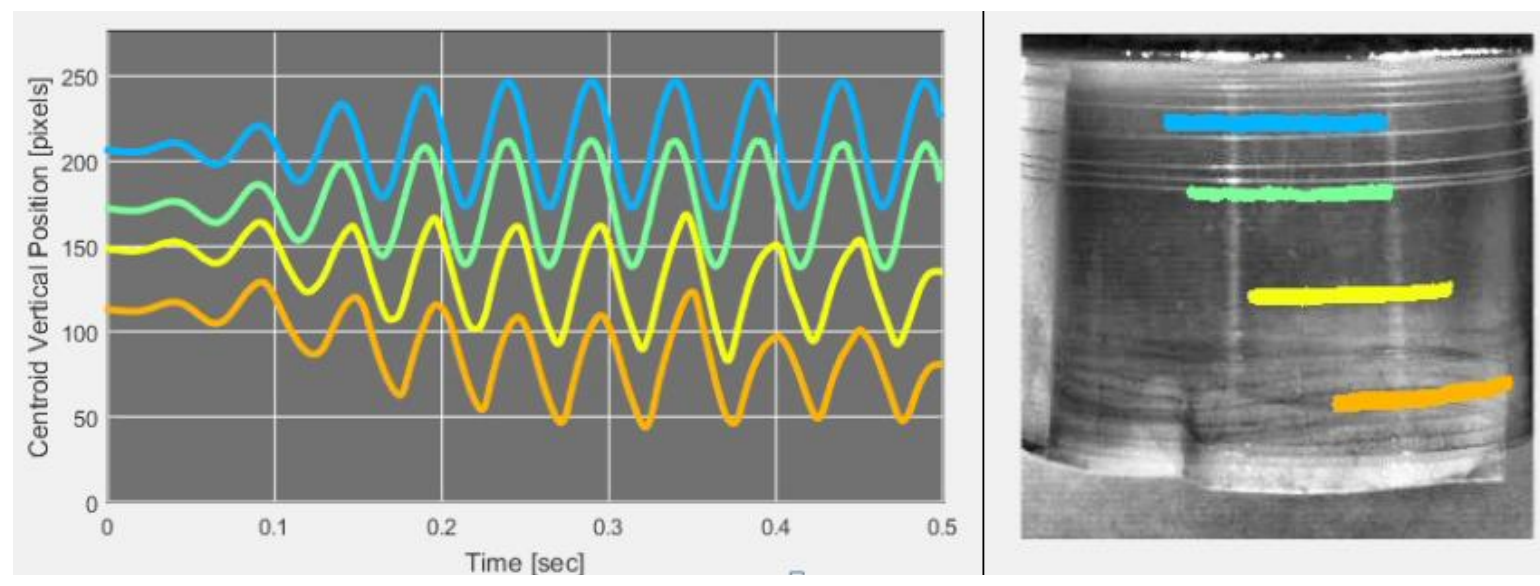

Figure 5. $20 \mathrm{~Hz}$ sinusoidal vibration test results of the clear mylar roll illustrating the vertical positions of the centroids of each marking as a function of time.

\section{III.B. Vibration of Kapton Polyimide Roll}

Using the vibration measurement technique presented in the previous section, we performed similar experiments on a higher fidelity vibration test model. Note that since most materials are not transparent, we utilized a roll of $0.1 \mathrm{~mm}$-thick Kapton polyimide which is slightly transparent, although in this case the inner layers are difficult to visualize when their number is large.

The test setup is shown in Figure 6. A 20 inch long, 1 inch wide strip of Kapton was wrapped around a circular hub of diameter 1.25 inches for a total of approximately five loops. The innermost layer was fastened directly to the hub, constraining the motion of the innermost layer to the motion of the hub. The outermost layer was attached to a mechanical extension spring, which provided a uniform $0.3 \mathrm{~N}$ tension force. This tension force maintained a consistent contact pressure between layers and prevented any major loosening of the wrapped roll over the course of the vibration test. The other end of the spring was attached to a 2 inch long screw, greatly facilitating the adjustment of the spring tension if necessary.
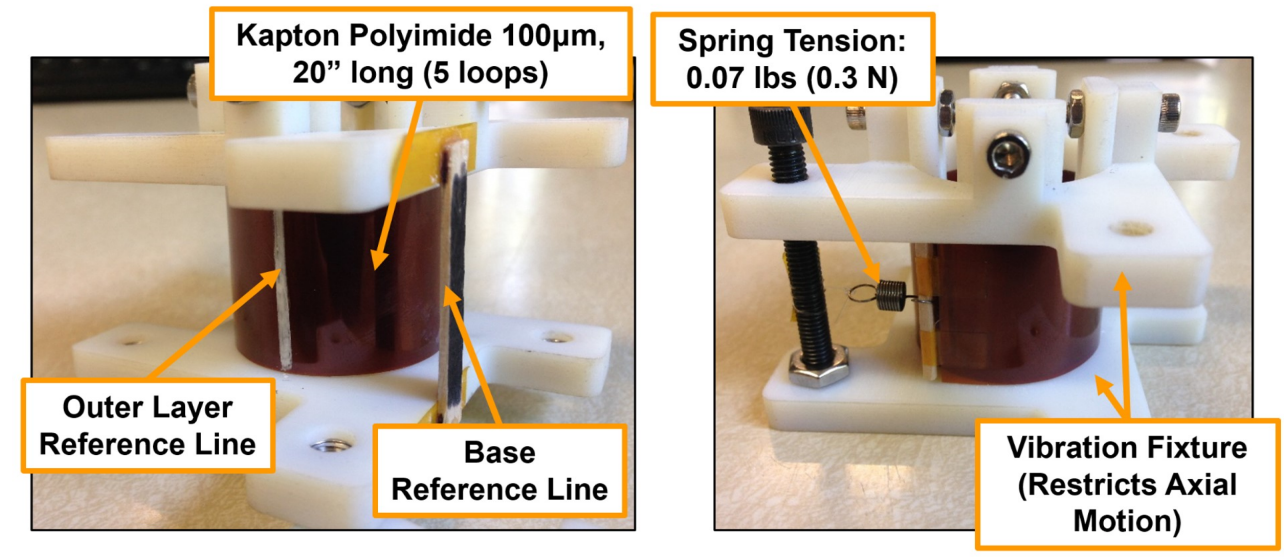

Figure 6. Kapton polyimide roll configuration.

The vibration fixture contains a flat section directly below and directly above the Kapton roll, restricting any axial motion of the roll. This is a more realistic configuration, since any unconstrained degrees of freedom may pose significant risks under a launch vibration environment. Since the innermost layer will have no motion relative to the vibration fixture hub and the outermost layer is attached to the vibration fixture via a mechanical spring, we expect the circumferential motion of the roll to be bounded above by the motion of the outermost layer. Hence, reference lines were added on the outermost layer and on the vibration fixture to provide visual indicators on the relative positions of these references, as shown in Figure 6 .

This higher fidelity vibration model was subjected to lateral-direction sinusoidal vibration using the setup shown in Figure 7. Similarly to the previous section, a laser vibrometer was used to measure the base 


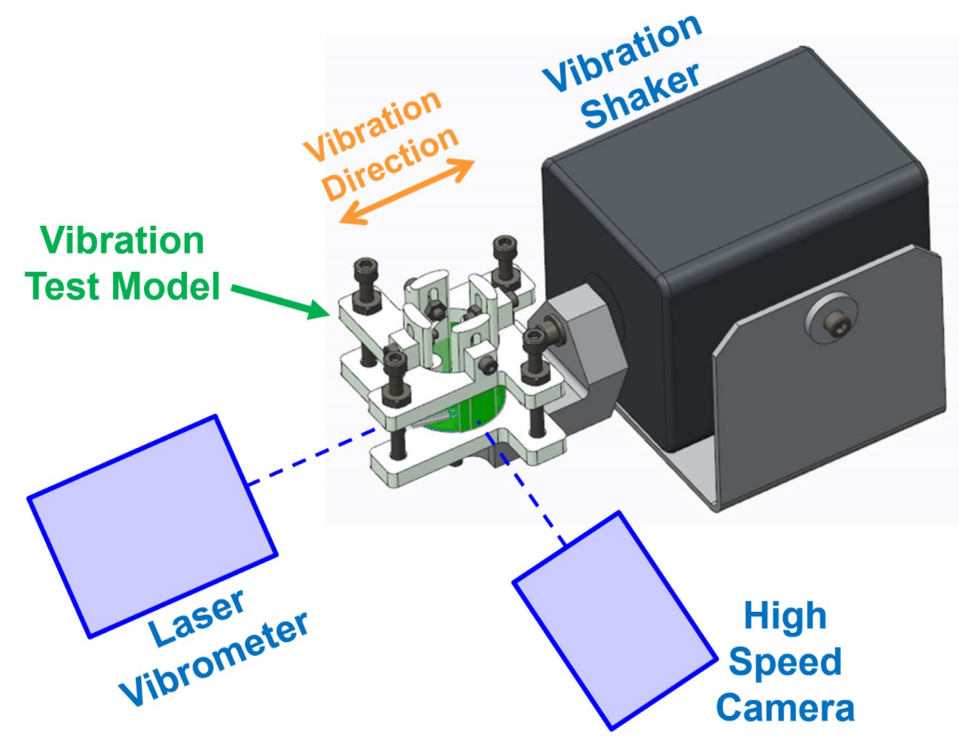

Figure 7. Vibration test setup for Kapton polyimide roll.

motion as a function of time and a high speed camera was used to capture the positions of the reference lines during the vibration test. The images were processed using the same methodology presented in the previous section, with only minor updates made to the custom mask to highlight the edges of the reference lines and the brightness criteria to extract the desired regions in the images.

The first $0.5 \mathrm{sec}$ of a $20 \mathrm{~Hz} 7.7 \mathrm{~g}$ peak acceleration sinusoidal vibration test are shown in Figure 8 . No significant relative displacements or phase delay between the outermost layer, in blue, and the base reference, in green, is easily observed from the recorded data. However, when normalized and plotted relative to the fixture base as shown in Figure 9, some relative motion is indeed observed. The RMS of the relative displacement between the outer layer and the fixture base was observed to be about $0.012 \mathrm{~mm}$. Since the relationship between the peak value and RMS value of a sinusoidal curve is a factor of $\sqrt{2}$, the estimated peak value is about $0.017 \mathrm{~mm}$. Using this information, we can then produce a simulation model (correlated to experimental data) that can be modified and used to predict the dynamic behavior of more complex configurations and at larger structural scales.
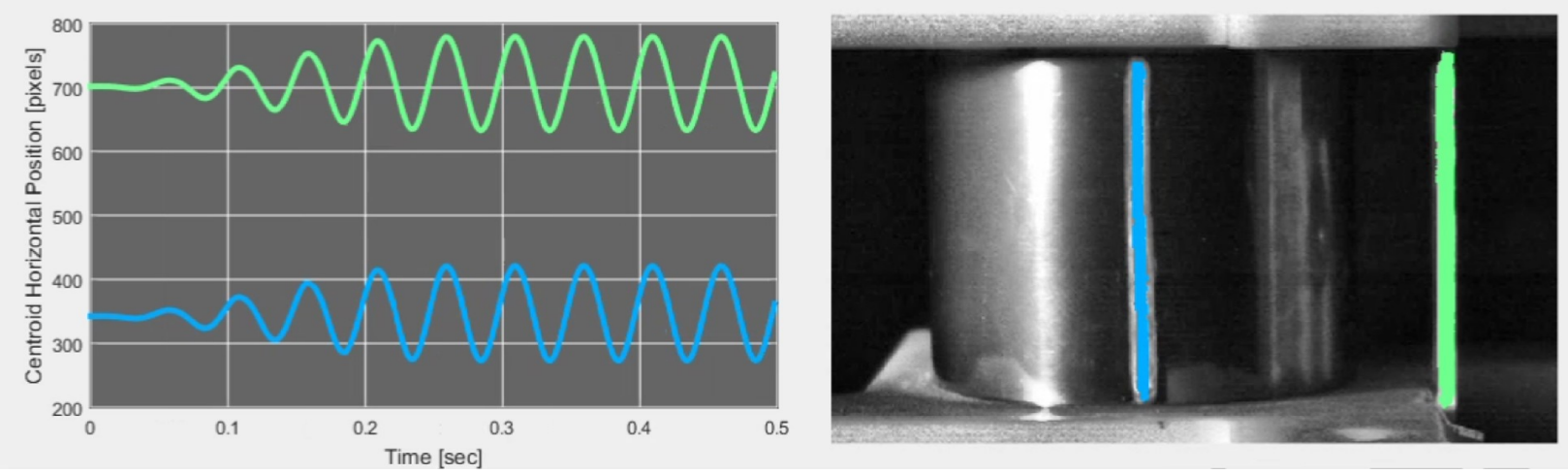

Figure 8. $20 \mathrm{~Hz}$ sinusoidal vibration test results of the Kapton polyimide roll illustrating the horizontal positions of the centroids of each reference line as a function of time. The blue line corresponds to the position of the outermost layer of the roll while the green line corresponds to the position of the vibration fixture base.

\section{Numerical Model}

In this section we present the development of a FEA model to simulate the wrapping and vibration of an ultralight structure. First we establish a validated simulation model, tuned to match the dynamic 


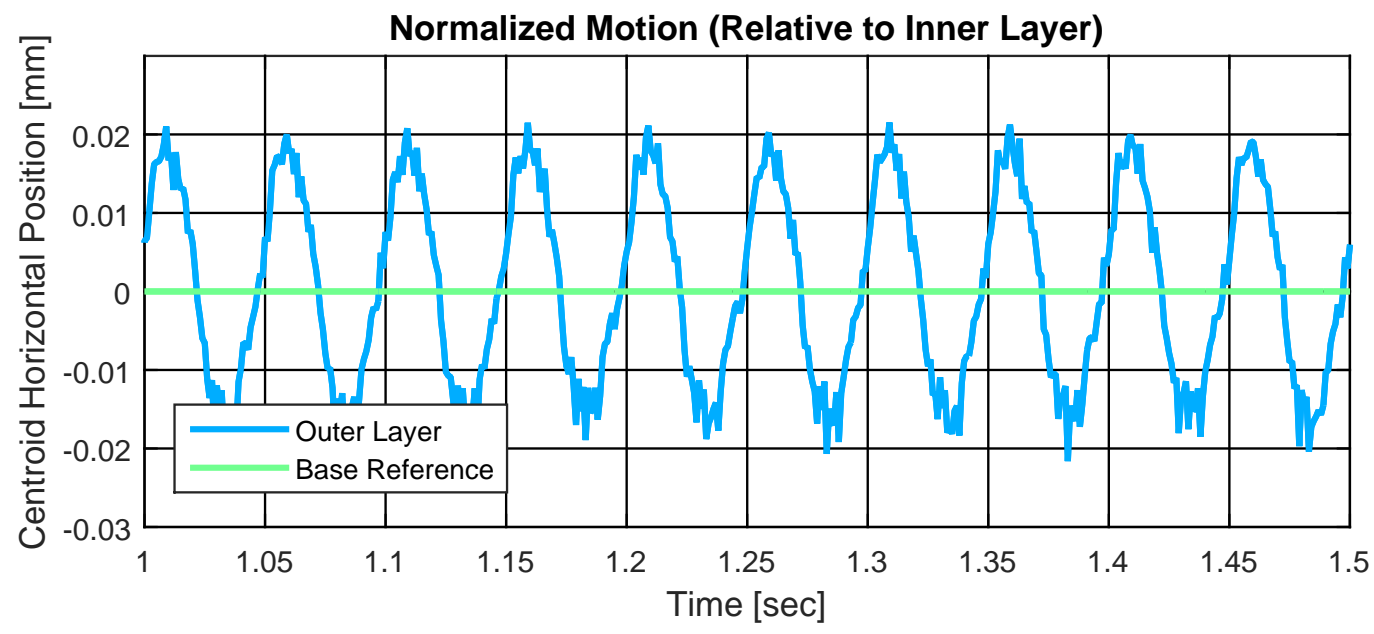

Figure 9. Relative motion between Kapton polyimide roll outer layer and vibration fixture base (which is attached to the innermost layer of the roll) during $20 \mathrm{~Hz}$ sinusoidal vibration testing.

responses of an associated experimental model. We then explore the vibration response of the model to various vibration input excitations. Lastly we present an analysis of a higher fidelity version of the ultralight wrapped structure, implementing the tuned parameters from the validated model.

\section{IV.A. Validation of Simulation Model Parameters}

For comparison with the experimental results from the previous section, an FEA model in LS-Dyna was developed to simulate a roll of Kapton polyimide wrapped around a circular hub. The hub is modeled as a discrete rigid cylinder using Belytschko-Tsay shell elements, as shown in Figure 10a. A single-layer Kapton strip was modeled with S/R Hughes-Liu shell elements. A line load at the end of the strip was included to represent the tension force applied by the mechanical spring in the experimental test model. The hub diameter, strip dimensions, and tension force in the simulation model were identical to those of the experimental model.

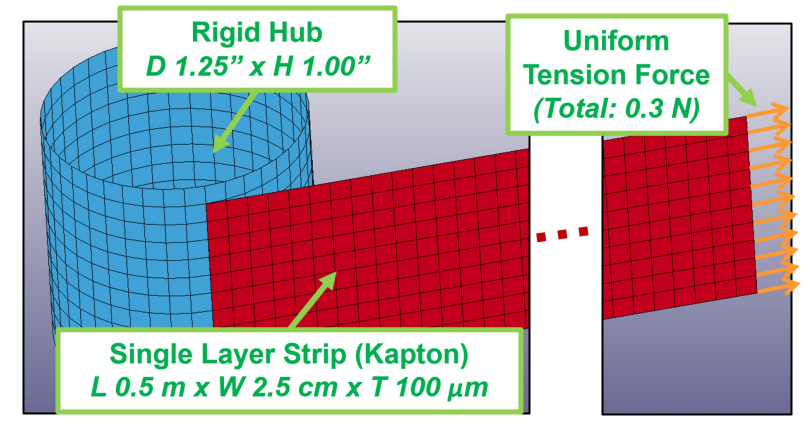

(a) FEA model configuration.

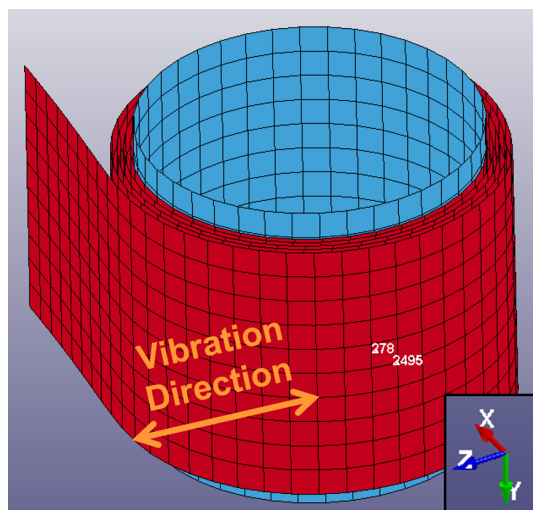

(b) Wrapped configuration.

Figure 10. LS-Dyna FEA simulation model of Kapton polyimide strip. In (b), two nodes of interest are highlighted: node 2495 on the outermost layer and node 278 on the rigid hub. The lateral direction of vibration is also indicated.

An automatic surface-to-surface contact constraint was applied between the hub and the strip. An automatic single surface contact constraint was defined for contact between the strip and itself, needed for the wrapping phase. To accurately represent the tangential friction behavior, a static-kinetic exponential decay routine was implemented, where the friction is equal to a static coefficient at zero slip rate, and exponentially decays to a kinetic coefficient as the slip rate increases. ${ }^{10}$ An arbitrary set of static and kinetic friction parameters were used in initial runs and successively tuned in later runs to produce results more consistent with the experimental results. To enhance stability of the wrapping simulation, the small contact 
penetration check option was turned on, as well as a soft constraint option using segment-based contact. To reduce transient oscillations during the simulation, mass-weighted and stiffness-weighted damping were applied on the strip.

A wrapping simulation similar to the simulation described in Section IV.C was performed to obtain an accurate contact pressure and residual strain distribution. The resulting wrapped configuration is shown in Figure 10b. To extract time history results, node 2495 was used to indicate the position of the outermost layer as a function of time while node 278 was used to indicate the position of the rigid hub.

After the wrapping phase, a brief period of stabilization was imposed, to damp out any residual transient vibrations and instabilities. Then, a sinusoidal vibration phase was imposed in a lateral direction as shown in Figure 10b with identical frequency and amplitude to the experimental model. Figure 11 illustrates the relative in-axis displacement between the strip outermost layer and the hub during the vibration phase of the simulation. It is clear that the tuning of the model was successful, as evidenced by the observed average peak relative displacement amplitude of about $0.017 \mathrm{~mm}$, which is the same as the value predicted by the experimental model. To achieve this tuning, it was observed that directly modifying the static and kinetic friction contact coefficients contributed the largest effect on the observed relative displacement. Modifying the damping parameters did not significantly affect the amplitude of the relative displacement, but rather introduced a higher-frequency transient oscillatory noise in the responses.

We noticed in this simulation a small shift towards the positive $\mathrm{Z}$ direction during the course of the run, indicating the outermost strip is gradually moving towards an increasingly wrapped state. This suggests that the input amplitude is not high enough to push the outermost strip towards a state of growing amplitude vibration or to produce any unwrapping of the roll, but rather only shakes out any looseness between adjacent strips throughout the roll to produce a more tightly wrapped configuration. This effect, which may be explained by the differences in the imposed boundary conditions, was not observed during the experiment. In the simulation, a constant $0.3 \mathrm{~N}$ line load was applied at the end of the strip, regardless of any relative motion of the end of the strip. However, in the experimental model, a mechanical spring with $0.3 \mathrm{~N}$ initial tension force is used. As the roll becomes increasingly wrapped during the vibration test, the strip end moves closer to the spring, reducing the overall spring deflection and hence decreasing the spring force and slowing down further wrapping. Conversely, as the roll is unwrapped during vibration, the strip end moves away from the spring, increasing the spring force and thus reducing further unwrapping.

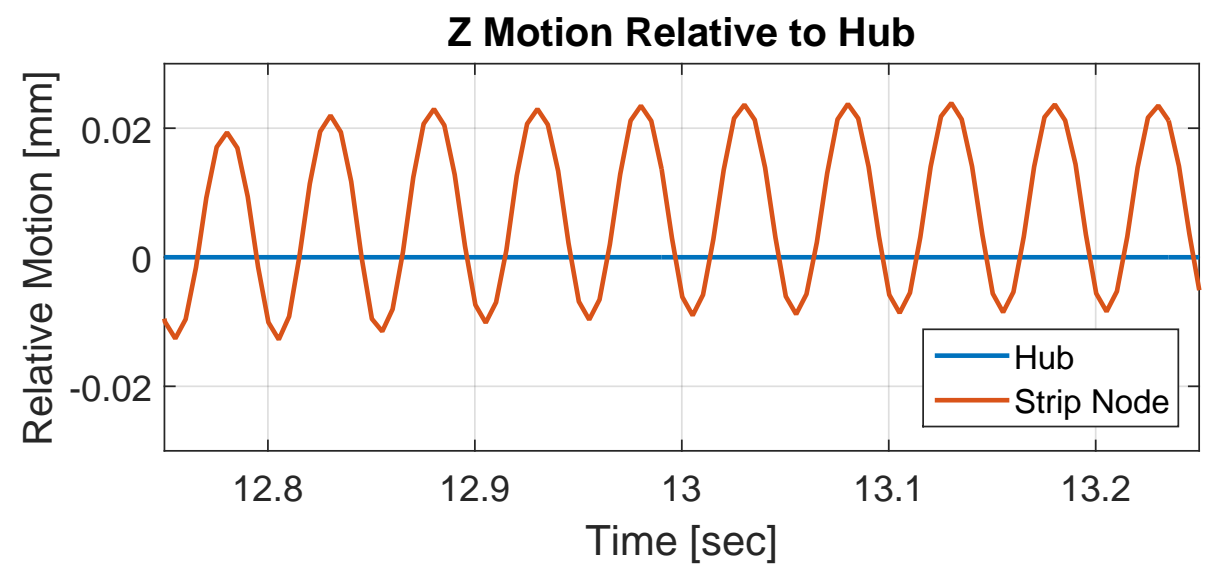

Figure 11. Normalized along-axis motion of outermost layer relative to hub during lateral sinusoidal vibration simulation.

Hence, we have shown that we can successfully develop a simulation model with parameters tuned to match the experimentally observed relative motion amplitudes. Using these parameters, we can explore the vibration response of the system to different vibration input excitations and also develop higher fidelity models that are more closely representative of the actual structures of interest.

\section{IV.B. Response to Other Vibration Input Excitations}

Using the tuned simulation model, we have explored the response of the same Kapton roll to other sinusoidal input excitation frequencies and amplitudes. 
For consistency with the previous tuned simulation, the final wrapped state (prior to any excitation) was subjected to various sinusoidal input amplitudes while using the same input frequency of $20 \mathrm{~Hz}$. Multiples of the original input amplitude $A_{0}=7.7 \mathrm{~g}$ peak acceleration were used. Identical boundary conditions were implemented, e.g. a $0.3 \mathrm{~N}$ line load at the end of the strip to represent the tension force applied by the spring in the experimental model, as shown previously in Figure 10a.

Figure 12 shows the relative motion between the outermost strip at Node 2495 and the fixed hub along the direction of vibration (i.e. the $\mathrm{Z}$ direction) for various input amplitudes. Refer again to Figure 10b for node location and vibration direction. At this node location, the $\mathrm{Z}$ motion also represents the circumferential motion around the hub, counteracted by the constant $0.3 \mathrm{~N}$ line load at the end of the strip, the friction between adjacent Kapton layers in the roll, and the tie constraint between the innermost strip layer end and the fixed hub. Note that in these simulations, a gradual ramp up to full level was applied for the 12.25-12.75 sec time range.

For input amplitudes of 1 and 2 times the nominal input amplitude, as shown in Figures 12a and 12b respectively, we observe a gradual positive $\mathrm{Z}$ shift over time, indicating the outermost strip is gradually moving towards an increasingly wrapped state. Again, this suggests that the input amplitude is not high enough to cause any unwrapping of the roll, but rather only shakes out any looseness between adjacent strips throughout the roll to produce a more tightly wrapped configuration. From 4 to 10 times the nominal input amplitude as shown in Figures 12c through 12f, we observe that not only the amplitude of relative motion is increased, but also the shift in the negative $\mathrm{Z}$ direction over time, which is indicative of increased unwrapping of the roll. This suggests that input amplitudes of this magnitude are high enough to induce unstable levels of vibration in the outermost strip layer and even in layers farther inward, causing increased gaps between layers and hence increased unwinding of the roll. This may be a result of either an insufficient line load force on the outermost strip, or insufficient friction between layers. Depending on the expected vibration loads for a configuration like this one, this information can be used to optimize the line load force to prevent any unstable unwrapping motion in future designs.

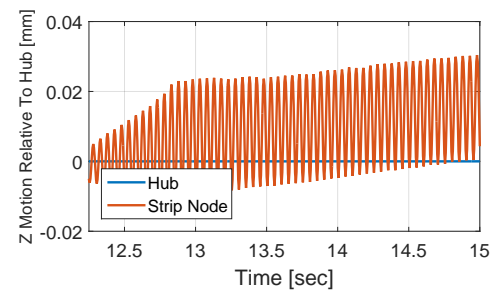

(a) $A=A_{0}$

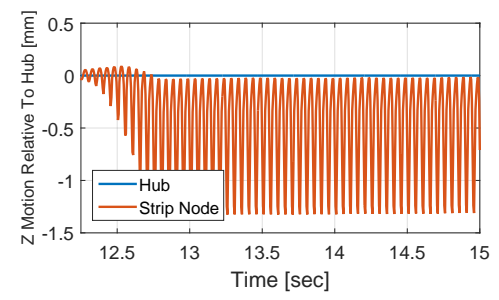

(d) $A=6^{*} A_{0}$

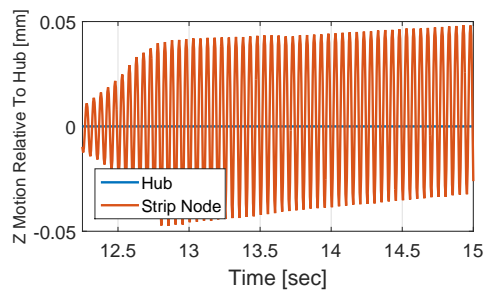

(b) $A=2^{*} A_{0}$

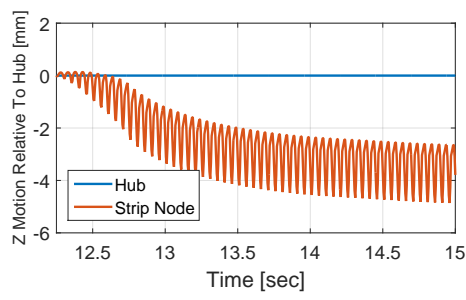

(e) $A=8^{*} A_{0}$

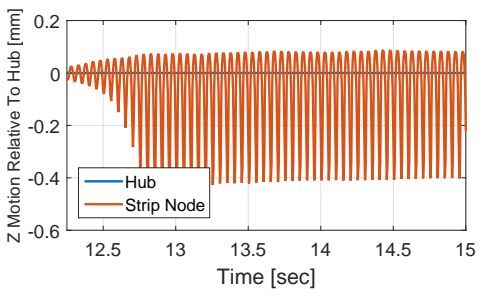

(c) $A=4^{*} A_{0}$

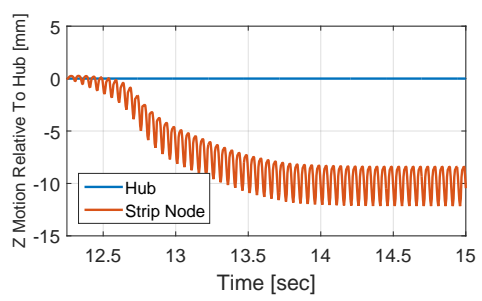

(f) $A=10^{*} A_{0}$

Figure 12. Relative motion in the $Z$ (i.e. circumferential) direction between the outermost strip and the fixed hub for various sinusoidal input amplitudes applied to the wrapped Kapton roll configuration shown in Figure 10b. Input amplitudes $A$ are multiples of the original input amplitude $A_{0}=7.7 \mathrm{~g}$ peak acceleration. Sinusoidal input frequency is $20 \mathrm{~Hz}$ for all cases.

Similar analyses were performed using sinusoidal input frequencies of $5 \mathrm{~Hz}, 10 \mathrm{~Hz}, 40 \mathrm{~Hz}$, and $100 \mathrm{~Hz}$, as shown in Figure 13. Similar results to in the $20 \mathrm{~Hz}$ input frequency case were obtained. Although not easily observed in Figure 13, for smaller amplitudes at each frequency, the relative $\mathrm{Z}$ motion is similar to that in Figures 12a and 12b, with fairly stable sinusoidal relative motion behavior and a gradual shift to an increasingly wrapped state. Similarly, for higher amplitudes at each frequency, the amplitude of relative motion is increased and the shift towards an unwrapped state are also observed. However, with increasing frequency, the required input amplitude to produce the unstable unwrapping trend is increased. For example, at $5 \mathrm{~Hz}$ input frequency (as shown in Figure 13a), the amplitude at which the unstable unwrapping trend is 
first observed is at $A=1^{*} A_{0}$, while at $100 \mathrm{~Hz}$ (as shown in Figure 13d), the required input amplitude is at $A=10^{*} A_{0}$.

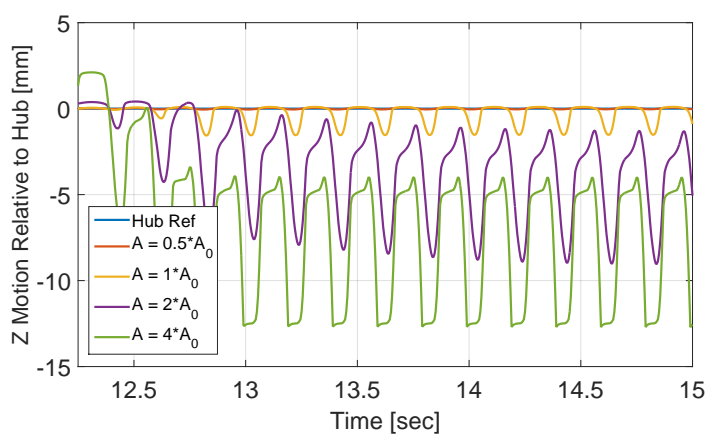

(a) $F=5 \mathrm{~Hz}$

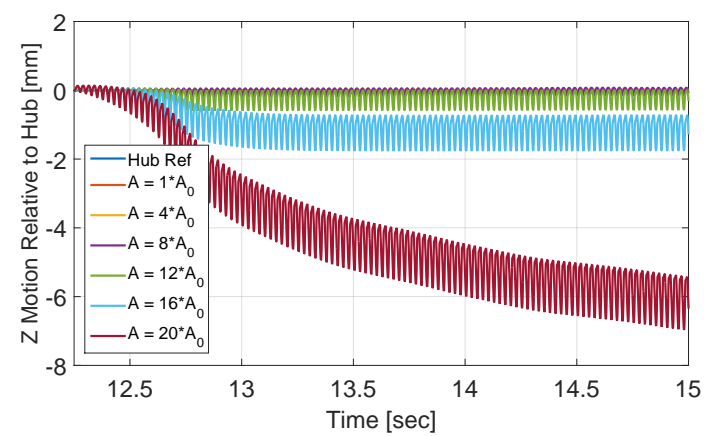

(c) $F=40 \mathrm{~Hz}$

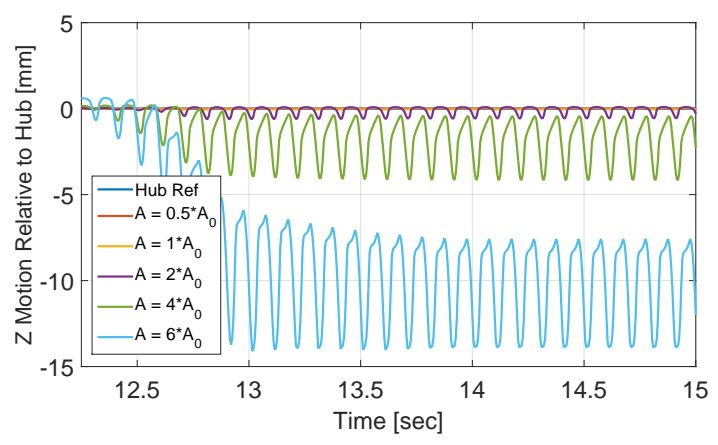

(b) $F=10 \mathrm{~Hz}$

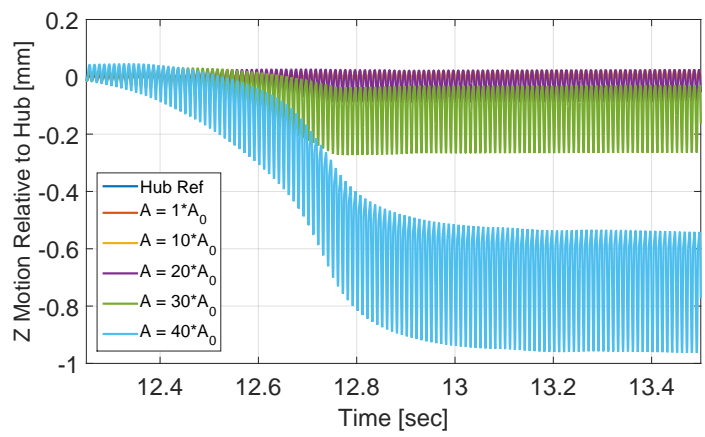

(d) $F=100 \mathrm{~Hz}$

Figure 13. Relative motion in the $\mathrm{Z}$ (i.e. circumferential) direction between the outermost strip and the fixed hub for various sinusoidal input frequencies and amplitudes applied to the wrapped Kapton roll configuration shown in Figure 10b. Input amplitudes $A$ are multiples of the original input amplitude $A_{0}=7.7 \mathrm{~g}$ peak acceleration.

The relative motion amplitudes and mean shifts for these cases are summarized in Figure 14 . The relative motion amplitude refers to the amplitude (i.e. (max value - min value)/2) of the $\mathrm{Z}$ motion of the outermost strip relative to the hub near the end of each vibration simulation run. This indicates the amount of oscillatory slippage between the outermost layer and the next inner layer during each cycle of vibration. The mean shift refers to the shift in the $\mathrm{Z}$ direction of the mean value of the relative motion over the course of each vibration simulation run, indicating the total amount of wrapping or unwrapping by the end of each case.

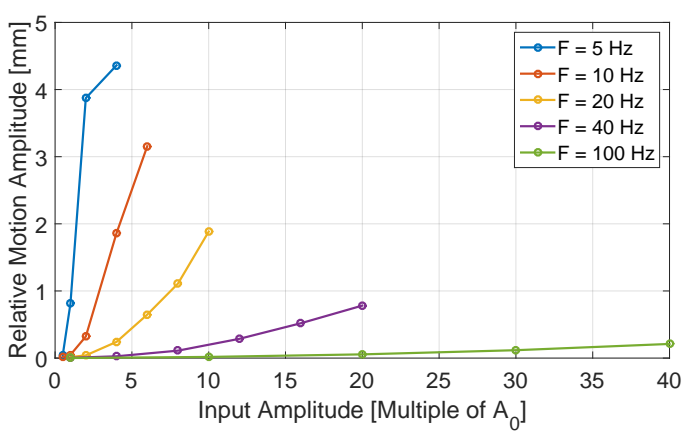

(a) Comparison of relative motion amplitudes

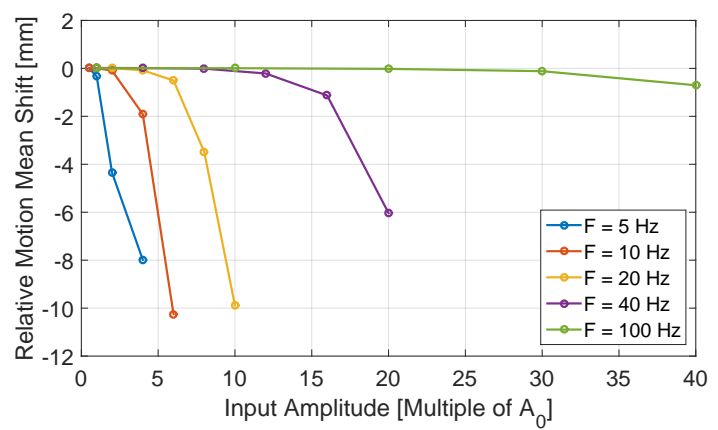

(b) Comparison of relative motion mean shifts

Figure 14. Summary of relative motion amplitudes and mean shifts in the $\mathrm{Z}$ (i.e. circumferential) direction between the outermost strip and the fixed hub.

As shown in Figure 14a, it appears that the higher the input frequency, the lower the relative motion amplitude. This intuitively makes sense however, since higher frequencies in general result in lower dis- 
placements for a given acceleration amplitude, so it is not unusual that the relative motion displacement amplitude is also decreased. In terms of the mean shift, shown in Figure 14b, it also appears that higher frequencies result in less shift. This may again be explained by the fact that higher frequencies produce lower relative displacements, which means less motion of the individual strip layers and hence less potential for unwrapping of the roll.

As shown through this exercise with various input excitation frequencies and levels, we have obtained an understanding of the limitations of the current constraint configuration as well as identified areas for improvement for future designs. For example, a higher line load (which represents a higher spring tension force) at the end of the outermost strip layer may be implemented to restrict motion under the higher acceleration input levels.

\section{IV.C. Higher Fidelity Numerical Model}

The SSPI packaging scheme ${ }^{1}$ envisages symmetric boundary conditions, with tension applied at both ends of the strip terminating and a split hub. We developed a higher fidelity numerical model in LS-Dyna using the same dimensions and parameters as in the validated single-strip simulation model. Rather than clamping one end of the strip to the hub, the hub is split into two teardrop shaped plugs and the strip is passed through the middle, as shown in Figure 15a. The outer radius of these plugs is $32 \mathrm{~mm}$, matching the previous simulations. The strip properties remain the same except for the length which has been doubled to $1 \mathrm{~m}$. The strip ends are constrained in $\mathrm{Y}$ and $\mathrm{Z}$ to move with the hub, and a tension line-load is applied in the $\mathrm{X}$ direction as in the previous simulations.

To wrap the strip, the plugs were rotated about the central axis of the hub until the fully coiled configuration in Figure 15b had been reached. With the strip coiled, a brief stabilization was applied to damp out residual vibrations. Then sinusoidal vibration in the $\mathrm{Z}$ direction was applied to the hub, at a frequency of $20 \mathrm{~Hz}$ with peak accelerations of $A_{0}=7.7 \mathrm{~g}$.

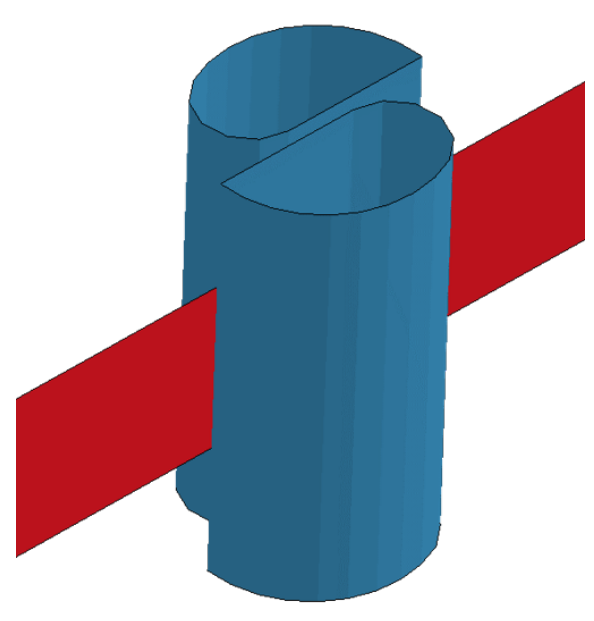

(a) Initial strip configuration, full strip length hidden for clarity

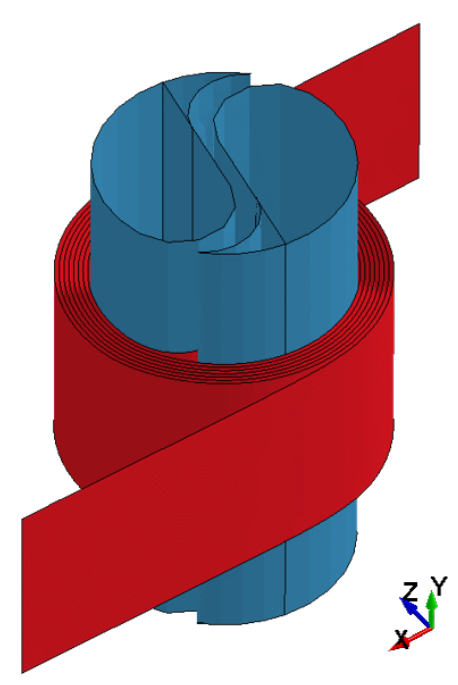

(b) Strip fully coiled and under $0.3 \mathrm{~N}$ tension

Figure 15. Wrapping of a single strip. Strip is shown in red and plugs in blue. The tips of the plugs are added during wrapping to provide a smooth cylindrical surface.

This higher fidelity model was used to answer the following three questions. (a) Would sinusoidal vibration in the $\mathrm{Z}$ direction cause the strip to move in the $\mathrm{Y}$ direction, now that the strip is not clamped directly to the hub? (b) Would the strip completely unwrap now that neither end is constrained in the X direction, or would it instead reach a steady slip state? (c) What effect does the tension force have on the slip behavior in both the circumferential and Y directions?

To answer these questions, two sets of simulations were performed. In the first set the strip was only constrained in the $\mathrm{Y}$ direction at the strip ends. In the second set displacement in the $\mathrm{Y}$ direction was constrained along the entire length of the strip. For both sets of simulations, the tension force was varied 
from the nominal $0.3 \mathrm{~N}$ up to $1.8 \mathrm{~N}$. The maximum slip in the circumferential and Y directions after $4.75 \mathrm{~s}$ of vibration is shown in Figures 16a and 16b as a function of force.

For the full Y constraint cases the circumferential slip decreases in a bi-linear fashion with respect to force, with the transition point between the two slopes at $0.8 \mathrm{~N}$. Up until this point, increasing the tension force has a significant effect on the circumferential slip between the layers. At $0.8 \mathrm{~N}$ all the layers move together with the hub. After this point there is a diminishing effect in increasing the force. For the other case, there is a combination of both circumferential and Y slip which results in lower total circumferential slip.

The separation of layers below $0.8 \mathrm{~N}$ has a clear effect on the maximum Y slip in the wrapped strip. When the force is below $0.8 \mathrm{~N}$ the layers separate every cycle which allows the strip to also move in the $\mathrm{Y}$ direction. Above $0.8 \mathrm{~N}$ static friction between the layers is sufficient to stop the strip moving in the $\mathrm{Y}$ direction. The small amount of slip that does occur happens at the very start of the applied vibration. For the case where the strip is constrained in the $\mathrm{Y}$ direction along its entire length, there is of course no slip in the $\mathrm{Y}$ direction. From this we conclude that the previous simulations was in the second regime, possibly since the strip was only $0.5 \mathrm{~m}$ long and was clamped directly to the hub.

There is a jump in the circumferential slip for the $0.3 \mathrm{~N}$ simulation. This is because the force is insufficient for the slip to reach an equilibrium, but continues to vary.

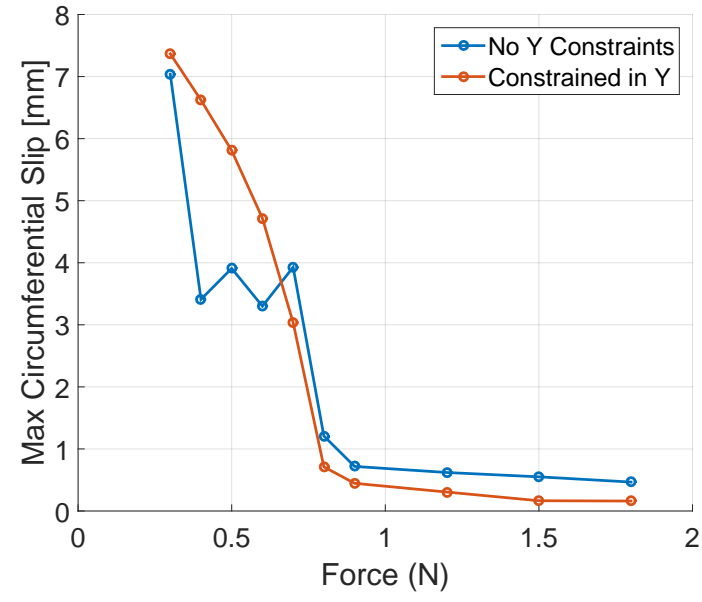

(a) Slip in circumferential direction for outermost strip layer as function of applied tension force. The blue line corresponds to constraints in the Y direction applied only at the ends, and the red line to $\mathrm{Y}$ constraint applied to the full length of the strip.

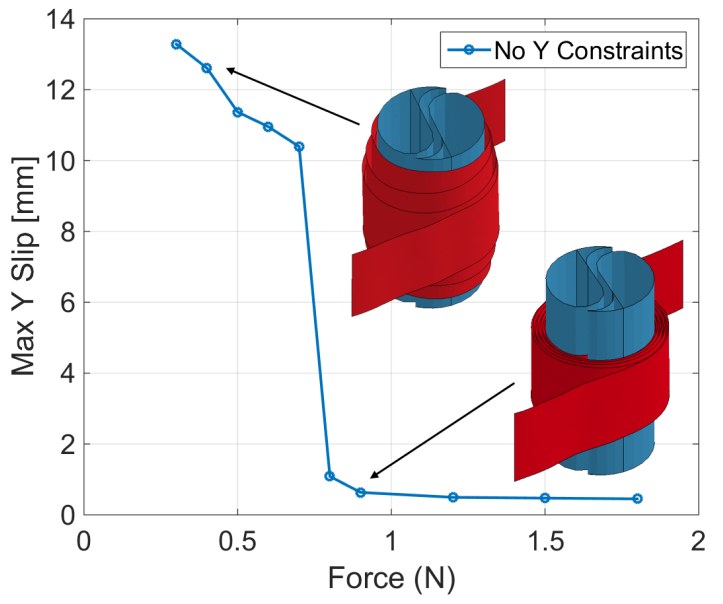

(b) Slip in Y direction as a function of applied tension force.

Figure 16.

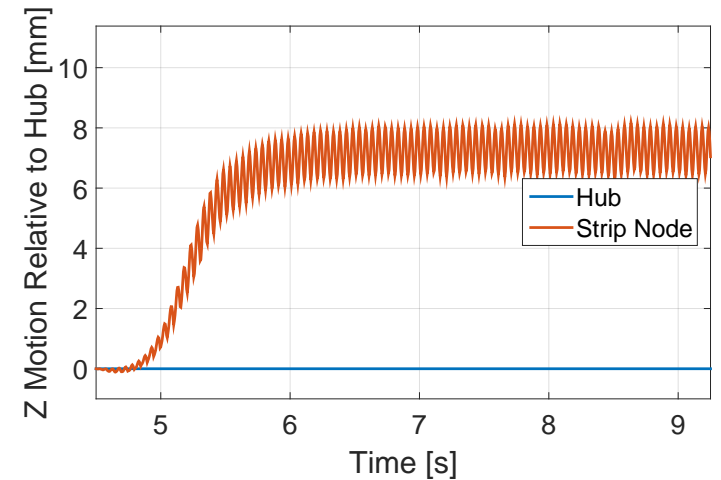

(a) Y constraints applied the full length of the strip, $0.3 \mathrm{~N}$ tension applied to the ends

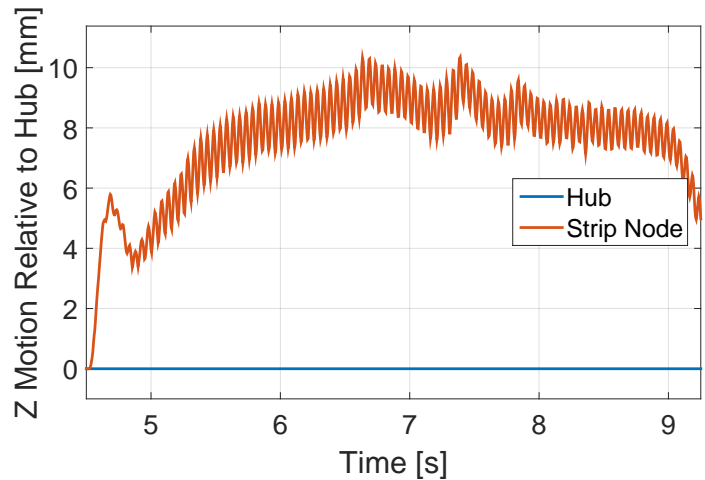

(b) $\mathrm{Y}$ constraints applied only to strip ends, $0.3 \mathrm{~N}$ tension applied to the ends

Figure 17. Circumferential slip of the outer layer over $4.75 \mathrm{~s}$ of vibration. 
Figures $17 \mathrm{a}$ and $17 \mathrm{~b}$ show the circumferential slip over time for the nominal $0.3 \mathrm{~N}$ case. When constrained in the $\mathrm{Y}$ direction, the circumferential slip reaches a steady value of about $8.5 \mathrm{~mm}$. When unconstrained, the continued slippage in the Y direction makes the circumferential slip far less stable, as shown in Figure 17b.

\section{Conclusion}

We presented a methodology to quantify the dynamic behavior of an ultralight wrapped spacecraft structure under a vibration environment, taking into account an estimation of the final shape and pressure distribution in the wrapped state. Results from a small-scale experimental model were used in the development of a numerical finite element model, taking into account non-linear effects such as contact forces and friction between adjacent layers.

We found that the circumferential slip of a wrapped strip increases with increased sinusoidal vibration amplitude and decreases with frequency. We also found that slip in both the direction parallel and perpendicular to vibration is bi-linear with respect to force. At forces below a critical transition force increasing force corresponds to significant decreases in slip. At the transition force however the wrapped strip vibrates together with the hub and increasing the force beyond this point results in diminishing returns.

This tuned model can provide an understanding of the model dynamics, final wrapped shape, and launch vibration survivability at different scales e.g. full-scale, thus providing valuable insight to aid in system design development and optimization efforts.

\section{Acknowledgments}

Financial support from Northrop Grumman Corporation is gratefully acknowledged.

\section{References}

\footnotetext{
${ }^{1}$ Arya, M., Lee, N., Pellegrino, S., "Ultralight Structures for Space Solar Power Satellites," 3rd AIAA Spacecraft Structures Conference, 2016.

${ }^{2}$ Stern, T., "Composite Beam Roll-Out Array Technology." 34th Space Power Workshop, Manhattan Beach, CA, USA, 2016, 18-21 April.

${ }^{3}$ White, S., "ISS ROSA Solar Array Flight Experiment." 34th Space Power Workshop, Manhattan Beach, CA, USA, 2016, 18-21 April.

${ }^{4}$ Stern, T., Steele, K., "Composite Beam Roll-Out Array- A Multifunctional Deployable Structure for Space Power Generation." 3rd AIAA Spacecraft Structures Conference, 2016.

${ }^{5}$ Spence, B., White, S., Douglas, M., "Elastically deployable panel structure solar arrays." U.S. Patent No. 9,156,568., 2015.

${ }^{6}$ Mori, O., Sawada, H., Funase, R., Endo, T., Morimoto, M., Yamamoto, T., Tsuda, Y., Kawakatsu, Y. and Kawaguchi, J., , "Development of first solar power sail demonstrator-IKAROS." 21st International Symposium on Space Flight Dynamics (ISSFD 2009), CNES, Toulouse, France., 2009.

${ }^{7}$ Tsuda, Y., Mori, O., Funase, R., Sawada, H., Yamamoto, T., Saiki, T., Endo, T. and Kawaguchi, J., "Flight status of IKAROS deep space solar sail demonstrator." Acta Astronautica, 69.9, 2011., pp 833-840.

${ }^{8}$ Mallikarachchi, H. M. Y. C., and S. Pellegrino. "Quasi-static folding and deployment of ultrathin composite tape-spring hinges." Journal of Spacecraft and Rockets, 48.1, 2011., pp 187-198.

${ }^{9}$ Cazzolato, B., Wildy, S., Codrington, J., Kotousov, A., and Schuessler, M. "Scanning laser vibrometer for non-contact three-dimensional displacement and strain measurements." Proceedings of the Australian Acoustical Society Conference, Geelong, Australia, Nov 24. Vol. 26. 2008.

${ }^{10}$ Oden, J., and Martins, J. "Models and computational methods for dynamic friction phenomena." Computer Methods in Applied Mechanics and Engineering, 52.1, 1985, pp. 527-634.
} 